\title{
Optical and microstructural investigations of porous silicon
}

\author{
R PRABAKARAN ${ }^{\ddagger}$, R KESAVAMOORTHY* and ALOK SINGH ${ }^{\dagger}$ \\ Materials Science Division, ${ }^{\ddagger}$ Physical Metallurgy Section, Indira Gandhi Centre for Atomic Research, Kalpakkam \\ 603 102, India \\ ${ }^{\dagger}$ Department of Physics, Loyola College, Chennai 600 034, India
}

MS received 12 October 2004; revised 9 February 2005

\begin{abstract}
Raman scattering and photoluminescence (PL) measurements on (100) oriented $n$-type crystalline silicon ( $c$-Si) and porous silicon (PS) samples were carried out. PS samples were prepared by anodic etching of $c$-Si under the illumination of light for different etching times of 30, 60 and $90 \mathrm{~min}$. Raman scattering from the optical phonon in PS showed the redshift of the phonon frequency, broadening and increased asymmetry of the Raman mode on increasing the etching time. Using the phonon confinement model, the average diameter of Si nanocrystallites has been estimated as $2.9,2.6$ and $2.3 \mathrm{~nm}$ for 30, 60 and 90 min samples, respectively. Similar size of Si crystallites has been confirmed from the high resolution transmission electron microscopy (HRTEM). Using 2TO phonon mode intensity, we conjectured that the disordered Si region around the pores present in $30 \mathrm{~min}$ PS dissolved on etching for $90 \mathrm{~min}$. The photoluminescence (PL) from PS increased in intensity and blue shifted with etching time from $2 \cdot 1-2 \cdot 3 \mathrm{eV}$. Blue shifting of PL is consistent with quantum confinement of electron in Si nanocrystallites and their sizes are estimated as $2 \cdot 4,2 \cdot 3$ and $2 \cdot 1 \mathrm{~nm}$ for 30,60 and $90 \mathrm{~min}$ PS, respectively which are smaller than the Raman estimated sizes due to temperature effect. Unambiguous dominance of quantum confinement effect is reported in these PS samples.
\end{abstract}

Keywords. Porous silicon; Raman scattering; photoluminescence; quantum confinement; high resolution transmission electron microscopy.

\section{Introduction}

Crystalline silicon $(c-\mathrm{Si})$ is the single most important material of the last century that has been the cornerstone of the semiconductor industry and has spearheaded extraordinary technological advancements. Bulk $c$-Si, however, has one major deficiency in that it has an indirect bandgap, making it unsuitable for integrating light with electronics (optoelectronics). Thus, $c$-Si has only very poor luminescence in the near infrared $(1100 \mathrm{~nm})$ region. As a result of this, solid-state light-emitting diodes (LEDs) are generally fabricated from direct band gap semiconductors such as GaAs, organic compounds and more recently nanostructured materials. These solutions not only make the integration of optoelectronic devices with conventional $\mathrm{Si}$ technology difficult but also expensive. It is against this background that the observation of intense visible luminescence in porous silicon (PS) layers, prepared by electrochemical etching, received widespread attention (Canham 1990, 1992; Cullis and Canham 1991).

The microstructural, chemical, electronic and optical properties of PS have been extensively studied by various experimental techniques (Bisi et al 2000; Prabakaran et al 2002, 2003, 2004, 2005). Owing to large variety of PS

*Author for correspondence (rkm@igcar.ernet.in) structures that can be produced by various techniques under different conditions, the experimental results often refer to very different PS structures. Porous silicon has many micropores and silicon residuals. The most important structural property of PS is that the single-crystalline feature of the substrate is retained even when the size or width of silicon residuals is extremely reduced during anodization. In many cases, the average diameter of crystallites is decreased down to the level of $2-3 \mathrm{~nm}$. This is below a critical size for strong quantum confinement effects, since the exciton Bohr radius in silicon is $4.3 \mathrm{~nm}$ (Yoffe 1993).

In the present work, PS samples were prepared by anodic etching of (100) oriented $n$-type $c$-Si under illumination of light for 30, 60 and 90 min etching times. These samples had small $\mathrm{Si}$ nanocrystallites in the porous layer. They did not have considerable amount of $a$-Si, disordered $\mathrm{Si}, \mathrm{SiH}_{x}$ and siloxene derivatives. Si nanocrystallite size in these PS samples was obtained from Raman scattering and PL using quantum confinement model.

\section{Experimental}

Phosphorous doped $n$-type (100) oriented $c$-Si (Metkem, India) having a resistivity of $8 \Omega$-cm was used to prepare PS at room temperature (RT) in an electrolytic etching bath of $1: 2$ mixture of HF (48\%) and ethanol (98\%) un- 
der a constant current density of $20 \mathrm{~mA} / \mathrm{cm}^{2}$. With platinum wire as cathode and silicon as anode, anodization was carried out in a plastic container under illumination provided by a $100 \mathrm{~W}$ halogen lamp placed at $45 \mathrm{~cm}$ away from the anode for 30, 60 and 90 min to obtain the PS of different porosities. Ethanol was preferred because of its ability to reduce the surface tension of the solution, allowing in that way to the generation of smaller structures. After anodization the samples were rinsed in de-ionized water and dried under a pressure of $10^{-3}$ mbar at RT. All samples were stored under ambient atmosphere for about 2 weeks and Raman, photoluminescence and HRTEM measurements were carried out.

High resolution transmission electron microscopy (HRTEM) study was carried out in a JEOL 2000 EX-II microscope using $200 \mathrm{keV}$ electron beam. For this analysis, samples were prepared by direct cleavage from the PS surface. PS sample surface was scraped with a sharp razor blade and the fragments were collected on a holey carbon coated $\mathrm{Cu}$ grid. The micrographs and the transmission electron diffraction patterns from these fragments were obtained.

The Raman and PL spectra of $c$-Si and PS were recorded at RT in a laser Raman spectrometer built around a double grating monochromator, SPEX model 14018. $488 \mathrm{~nm}$ line of Ar-ion laser was used as the source, which has a penetration depth of about $100 \mathrm{~nm}$ in $c$-Si. This beam of $\sim 60 \mathrm{~mW}$ was focused on the sample to $100 \mu \mathrm{m}$ spot and the scattered light in the backscattering geometry was collected using a camera lens (Nikkon) and a focusing lens. A thermoelectrically cooled photomulti- plier tube, model ITT-FW 130, was used to detect the scattered light after passing through the monochromator. A microprocessor based automated data collection system was used to record the spectra. A slit width of the monochromator corresponding to $4.4 \mathrm{~cm}^{-1}$ instrument resolution was employed for optical Raman spectra and PL. The scattered light was integrated for $10 \mathrm{~s}$ and digitally recorded at $0.5 \mathrm{~cm}^{-1}$ interval for Raman spectra and $10 \mathrm{~cm}^{-1}$ interval for PL spectra. On shining the laser beam, PL intensity decreased and reached a steady value in about 20 min after which Raman and PL spectra were recorded from the same spot on the sample. The sample surface was homogeneous in the sense that almost same results were produced at various spots.

\section{Results and discussion}

\subsection{Microstructure of PS from HRTEM}

Figure 1 shows a high resolution transmission electron bright field image along (110) axis of Si fragments obtained by scraping the surface of the 30 min etched PS. It shows a network structure with pores in-between. This solid network comprises of "finger" like structures of about 5-8 nm thick without any preferred direction. Inside these "fingers" a few nm thick $c$-Si grains are observed as lattice fringes of (111) planes surrounded by a thin amorphous phase of one or two lattice spacing $(a$ $\mathrm{Si}$ ). These silicon nanocrystallites are often elongated in shape. This microstructure is similar to those reported earlier (Berbezier and Halimaoui 1993). The sizes of the

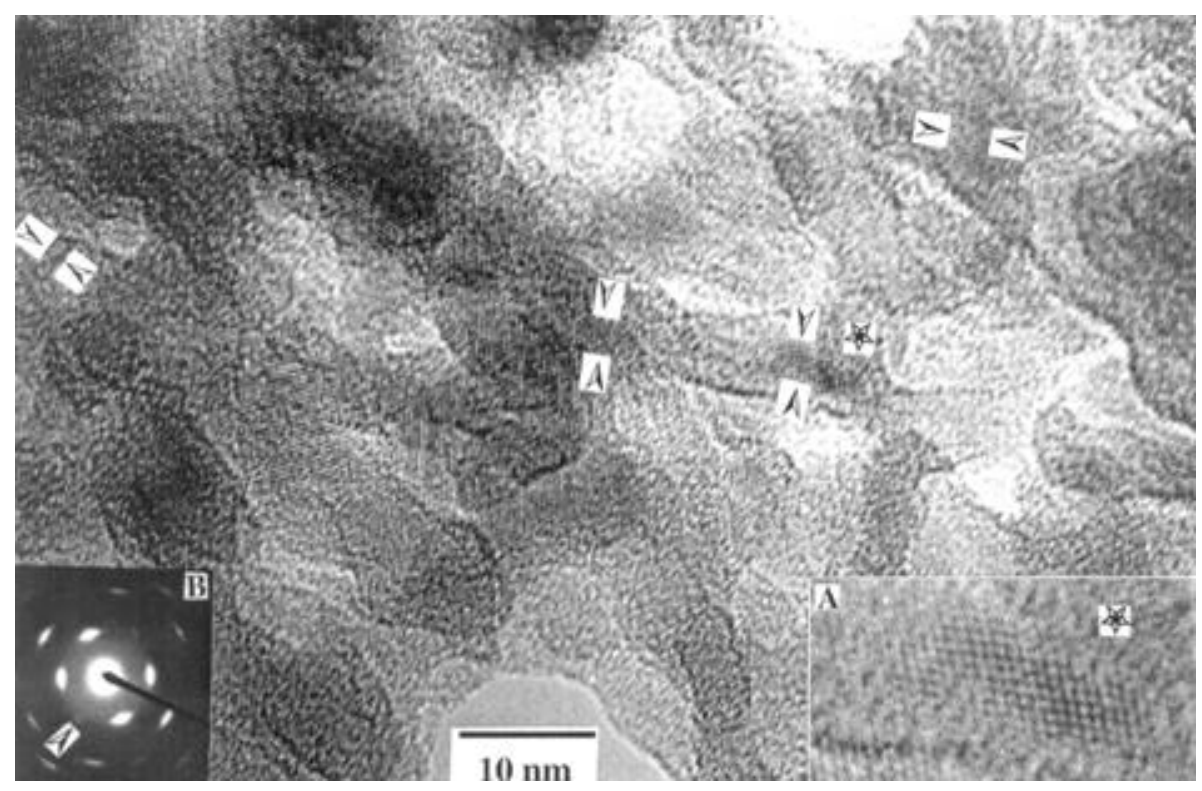

Figure 1. High resolution transmission electron micrograph of fragments from the surface of the 30 min etched PS sample. Inset A is the magnified picture of a spot (w) in the micrograph. Inset $\mathrm{B}$ is the TED pattern corresponding to the bright field image. Si nanocrystallites of $2-3 \mathrm{~nm}$ sizes are indicated by the arrow mark. 
silicon nanocrystallites vary from $1-8 \mathrm{~nm}$. This size range agrees with the estimated size using XRD pattern (Prabakaran et al 2002). Some of these particles of about 2$3 \mathrm{~nm}$ thick are indicated by arrows in figure 1 . The inset A shows the magnified view of a $\mathrm{Si}$ nanocrystallite of about $3 \mathrm{~nm}$ size. The larger silicon particles often contain planar defects breaking the continuity of the lattice. From this micrograph, it is difficult to estimate the mean size of the silicon nanocrystallites (which are all in the same crystallographic orientation) since they could not always be distinguished. The inset B shows the transmission electron diffraction (TED) pattern corresponding to this micrograph. This was taken from an area of about $1 \mu \mathrm{m}$ diameter. It shows arc-shaped diffraction spots, which indicate that the orientation of the $c$-Si particles deviates up to $\pm 14^{\circ}$ from the original single crystal lattice. The two sets of (111) spots corresponding to interplanar spacing of $0.312 \mathrm{~nm}$ are seen very prominently, as expected. In addition, a weak intensity spot is observed at (200) position, which is marked by an arrowhead in inset B. This forbidden reflection has also been observed very weakly in XRD pattern also (Prabakaran et al 2002). Diffuse ring of amorphous silicon was not observed clearly. The percentage of $a$-Si might be very small. This is consistent with the results of Raman spectra, which do not require the Raman intensity from $a$-Si (vide infra). A diffuse intensity corresponding to about $0.35-0.45 \mathrm{~nm}$ is observed in all our TED patterns. A diffuse peak centred at $0.39 \mathrm{~nm}$ with FWHM of $0.5 \mathrm{~nm}$ corresponding to $a$ $\mathrm{SiO}_{2}$ is observed around $22.5^{\circ} 2 \theta$ with FWHM of $8^{\circ} 2 \theta$ in XRD pattern (Prabakaran et al 2002). This is within the region of diffused intensity observed in the TED pattern and hence this is attributed to $a-\mathrm{SiO}_{2}$.

\subsection{Estimation of Si nanocrystallite diameter $(L)$ using Raman scattering}

Figure 2 shows Raman spectra from LO phonon for 30, 60 and $90 \mathrm{~min}$ etched PS in the range from $420-560 \mathrm{~cm}^{-1}$. Inset in this figure shows a narrow and symmetrical LO mode of $c$-Si. Since $c$-Si is (100) cut, LO mode is allowed and TO mode is forbidden. A Lorentzian line shape was fitted to the Raman spectrum of $c$-Si. The fit was very good and the peak position $\left(\omega_{\mathrm{LO}}\right)$ and the full width at half maximum $\left(\Gamma_{\mathrm{c}}\right)$ were obtained as $521.6 \mathrm{~cm}^{-1}$ and $5.5 \mathrm{~cm}^{-1}$, respectively. Crystalline quality of $c-\mathrm{Si}$ is very good since $\Gamma_{\mathrm{c}}$ is close to the instrumental resolution $\left(4.4 \mathrm{~cm}^{-1}\right)$. It is to be noted that the LO mode of PS broadens, becomes asymmetric and redshifts in comparison with that of $c$-Si. Si nanocrystallites relax the wavevector selection rule, which causes the Raman spectrum to broaden, redshift and become asymmetric (Sui et al 1992; Lee et al 1995).

Anodic etching of $c$-Si creates a nanocrystalline skeleton immersed in a network of pores. This complicated large internal surface area is partially oxidized at ambient, which results in the formation of smaller Si nanocrystallites. Richter et al (1981) considered these nanocrystallites to be spherical for explaining the phonon spectra. Later, Campbell and Fauchet (1986) invoked the same model and emphasized the significant differences between spherical, columnar and thin slab nanocrystals. HRTEM bright field image (figure 1) shows that the Si nanocrystallites are nearly spherical in shape and not of columnar or slab. In the present context, a strong phonon confinement model has been used to estimate the average diameter of the $\mathrm{Si}$ nanocrystallites. For spherical nanocrystallites, the first-order Raman line shape for LO phonon is given by (Xu et al 1999)

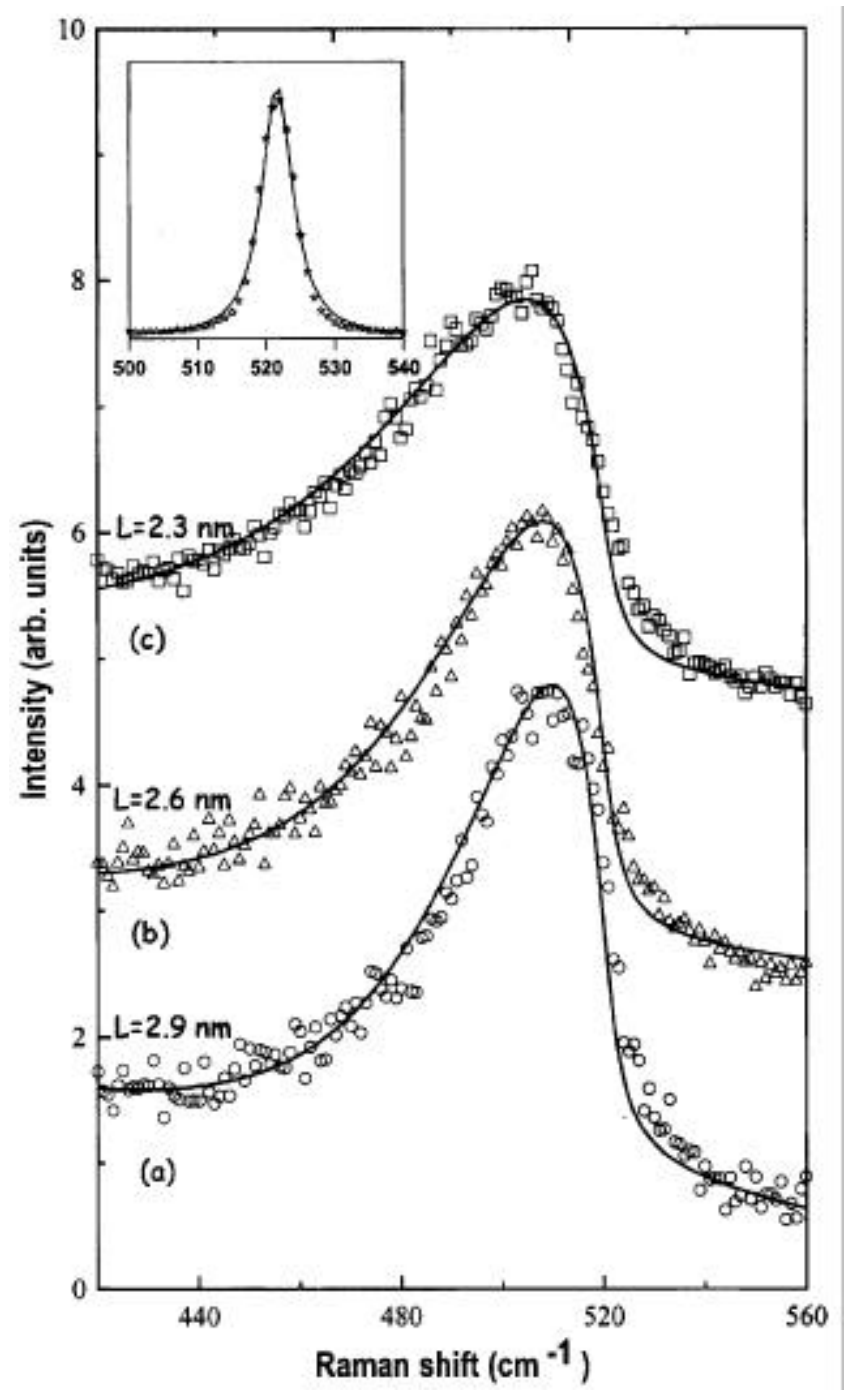

Figure 2. LO phonon Raman spectra of PS etched for (a) 30, (b) 60 and (c) $90 \mathrm{~min}$. The symbols are the experimental data. The solid lines are the generated spectra using quantum confinement model. The estimated size of the Si nanocrystallite is marked near each curve. The curves are shifted along the intensity axis for clarity. The inset shows the LO phonon Raman spectrum of $c$-Si. The symbols in the inset are the experimental data and the solid line is the Lorentzian fit. 


$$
I_{\mathrm{LO}}(\omega)=\int_{0}^{1} \frac{4 \pi q^{2}|c(0, q)|^{2}}{\left[\omega-\omega_{\mathrm{LO}}(q)\right]^{2}+\left[0 \cdot 5 \Gamma_{\mathrm{c}}\right]^{2}} \mathrm{~d} q
$$

where

$$
|c(0, q)|^{2}=\exp \left(\frac{-q^{2} L^{2}}{4 a^{2}}\right)
$$

and wavevector $q$ is expressed in the units of $2 \pi / a(a=c$ Si lattice spacing, $0.54 \mathrm{~nm}), L$ the diameter of the Si nanocrystallite, $\omega_{\mathrm{LO}}(q)$ the longitudinal phonon frequency at $q$ along [100] direction and $\Gamma_{\mathrm{c}}$ the FWHM of the crystalline silicon $\left(5 \cdot 5 \mathrm{~cm}^{-1}\right)$.

The phenomenological expression obtained from the reported dispersion curve for LO phonon branch along [100] direction is (Tubino et al 1972)

$$
\omega_{\mathrm{LO}}^{2}(q)=A+B \cos \left(\frac{\pi q}{2}\right)
$$

where, $A=1.7207 \times 10^{5} \mathrm{~cm}^{-2}$ and $B=1.0 \times 10^{5} \mathrm{~cm}^{-2}$. Equation (2) gives $\omega_{\mathrm{LO}}$ at $q=0$ as $521.6 \mathrm{~cm}^{-1}$ which matches well with our LO mode frequency in $c$-Si. The resultant intensity of the generated Raman spectrum can be obtained by

$$
I_{\mathrm{g}}(\omega)=P I_{\mathrm{LO}}(\omega)+Q \omega+R
$$

Here, $P$ is the coefficient which depends on the LO phonon contribution from $\mathrm{Si}$ nanocrystallites. $Q \omega+R$ in (3) represents background.

Using (3), the Raman spectra of PS were generated and fitted to the experimental Raman spectra as shown in figure 2. $L, P, Q$ and $R$ were the parameters obtained from the best fit to the experiment. As seen from figure 2, the fit was very good. This fit did not require the necessity to include the broad Raman mode at $480 \mathrm{~cm}^{-1}$ from amorphous silicon $(a-\mathrm{Si})$ or the contribution from defects induced TO mode. Since the Raman intensity from the LO mode at frequencies from $460-520 \mathrm{~cm}^{-1}$ is quite large, the small addition of intensity around $480 \mathrm{~cm}^{-1}$ from $a-S i$ might not be significant. The $c$-Si nanocrystallites diameter obtained from the best fit to figures $2 \mathrm{a}, \mathrm{b}$ and $c$ were $2 \cdot 9,2 \cdot 6$ and $2 \cdot 3 \mathrm{~nm}$ for 30,60 and $90 \mathrm{~min}$ etched PS, respectively. The values of $L$ and Raman peak positions
(PP) are listed in table 1. If the surface of PS contains the $\mathrm{Si}$ nanowires of nanodiameter and submicron length, the phonons would be confined in 2D. Sood et al (1992) used 2D integration over the LO phonon branch of $c$-Si to fit their Raman spectra of PS. We tried to fit our experimental Raman spectra with the Raman spectra generated by 2D integration over LO phonon branch but did not get a good fit. We included $a$-Si contribution also, but the good fit required negligible $a$-Si contribution. We included the contribution from defect induced TO branch, but obtained a good fit for negligible TO branch contribution. Hence, we believe that $c$-Si nanospheres are present in the PS samples studied here. This is corroborated by HRTEM (figure 1). Non-requirement of $a$-Si contribution to the Raman intensity is consistent with the XRD (Prabakaran et al 2004, 2005) and HRTEM (figure 1, inset B) results. Amorphous $\mathrm{SiO}_{2}$ does not have Raman contribution in the range from $420-560 \mathrm{~cm}^{-1}$.

\subsection{Defect induced Raman modes}

Figures $3 \mathrm{a}$ and $\mathrm{b}$ show the Raman spectra for 30 and $60 \mathrm{~min}$ etched PS in the range of $800-1050 \mathrm{~cm}^{-1}$, respectively. They show a peak around $930 \mathrm{~cm}^{-1}$ which is due to disorder induced $2 \mathrm{TO}$ phonon overtones (Edelberg et al 1997; Gregora et al 1994). This was fitted to Gaussian lineshape to obtain the peak position and FWHM. $30 \mathrm{~min}$ PS showed this $2 \mathrm{TO}$ phonon overtone at $936 \mathrm{~cm}^{-1}$ with $99 \mathrm{~cm}^{-1}$ FWHM while 60 min PS showed it at $933 \mathrm{~cm}^{-1}$ with $92 \mathrm{~cm}^{-1}$ FWHM. The area under the peak reduced considerably from 30-60 min sample. This mode was barely seen in 90 min sample and hence was not analysed. The intensity of the $2 \mathrm{TO}$ phonon mode was about 40 times lower than that of LO phonon in 30 min etched PS. This mode arises due to the disorder present in PS around the pores during their formation. During etching, the dissolution process results in a layer of disordered $a$-Si immediately around the pore. Well away from the pore, $c-\mathrm{Si}$ is present. The possible disordered Si layer would result in TO as well as $2 \mathrm{TO}$ mode appearance. Since TO mode was weak and fell in the region of high intense LO mode, it could not be resolved. However, 2TO phonon mode was observed in figure 3. As etching time increases, the disordered silicon layer around the pores dissolved and

Table 1. LO Raman mode peak position and Si nanocrystallite diameter $(L)$ along with PL emission peak positions, band widths, area and Si nanocrystallite diameter for various PS samples.

\begin{tabular}{lcccccc}
\hline $\begin{array}{l}\text { Etching time } \\
(\mathrm{min})\end{array}$ & $\begin{array}{c}\text { Raman PP } \\
\left(\mathrm{cm}^{-1}\right)\end{array}$ & $\begin{array}{c}L \text { from Raman } \\
(\mathrm{nm})\end{array}$ & $\begin{array}{c}\text { PL PP } \\
(\mathrm{eV})\end{array}$ & $\begin{array}{c}\text { PL FWHM } \\
(\mathrm{eV})\end{array}$ & $\begin{array}{c}\text { PL area } \\
(\text { a.u. })\end{array}$ & $\begin{array}{c}L \text { from PL } \\
(\mathrm{nm})\end{array}$ \\
\hline $0-(c-\mathrm{Si})$ & $521 \cdot 6(1)$ & - & - & - & - & - \\
30 & $508 \cdot 5(3)$ & $2 \cdot 9(1)$ & $2 \cdot 10(1)$ & $0 \cdot 34(1)$ & 3 & $2 \cdot 40(3)$ \\
60 & $506 \cdot 3(3)$ & $2 \cdot 6(1)$ & $2 \cdot 21(1)$ & $0 \cdot 30(1)$ & 23 & $2 \cdot 30(3)$ \\
90 & $503 \cdot 7(4)$ & $2 \cdot 3(1)$ & $2 \cdot 31(1)$ & $0 \cdot 43(1)$ & 165 & $2 \cdot 14(3)$ \\
\hline
\end{tabular}


hence the $2 \mathrm{TO}$ mode intensity decreased with increasing etching time. It is noted that the spectra in figure 2 have a width of about $40 \mathrm{~cm}^{-1}$, which is due to single phonon process while those in figure 3 have a width of about $90 \mathrm{~cm}^{-1}$, which is due to 2-phonon process. Figure $3 \mathrm{c}$ shows the Raman spectra of 30 min etched PS from 240 $350 \mathrm{~cm}^{-1}$. This was fitted to a Gaussian lineshape with $291 \mathrm{~cm}^{-1}$ peak position and $43 \mathrm{~cm}^{-1}$ FWHM. This peak arises due to the defect induced transverse acoustic phonon of $c$-Si (Gregora et al 1994; Edelberg et al 1997). This mode intensity was very low for $60 \mathrm{~min}$ PS and disappeared in $90 \mathrm{~min}$ PS. This is consistent with our suggestion that the disordered silicon layer dissolves on increasing the etching time to $90 \mathrm{~min}$.

\subsection{Photoluminescence in PS}

Figure 4 shows the photoluminescence spectra of the $c$-Si and PS etched for 30, 60 and $90 \mathrm{~min}$. The $c$-Si did not

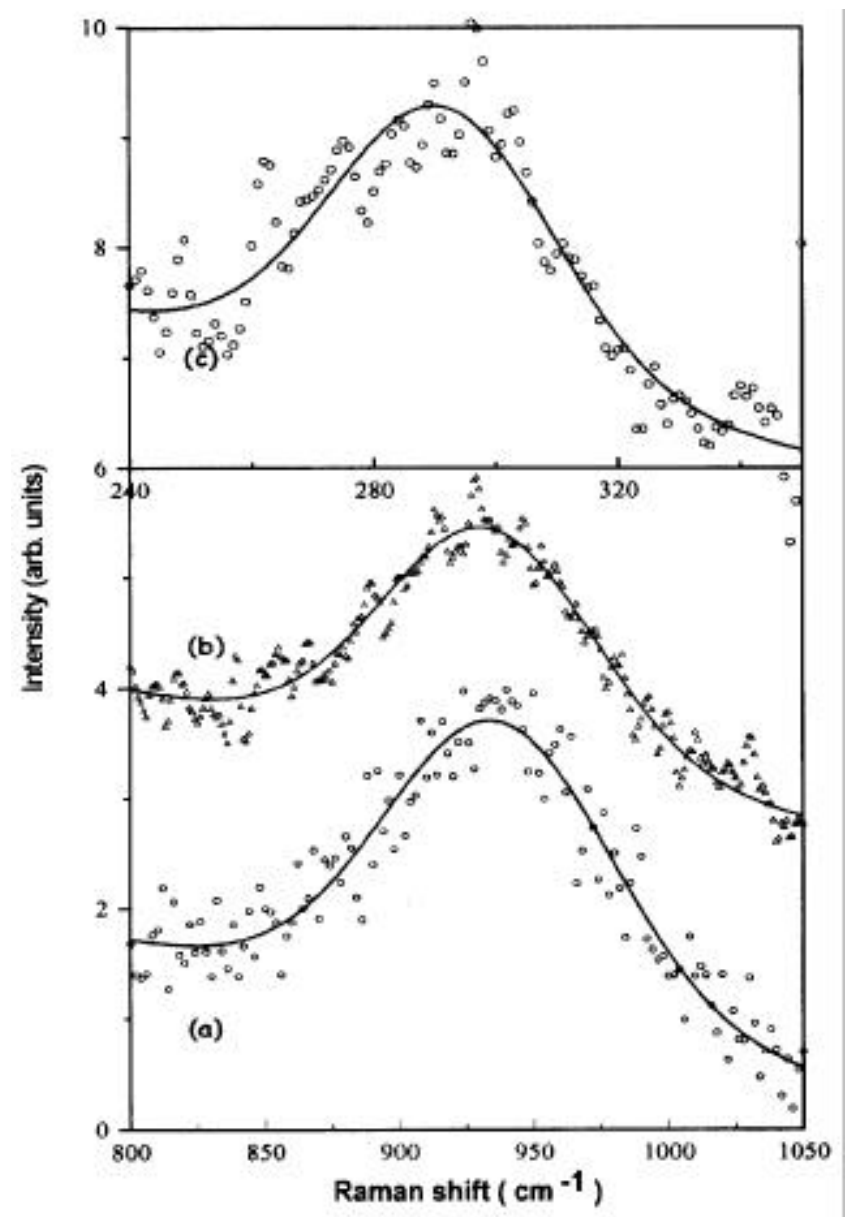

Figure 3. Raman spectra of (a) 30 and (b) 60 min etched PS samples in the range of $800-1050 \mathrm{~cm}^{-1}$. (c) Raman spectrum of $30 \mathrm{~min}$ etched PS sample in the range of $240-350 \mathrm{~cm}^{-1}$. The continuous curve is the Gaussian fit. The curves are shifted along the intensity axis for clarity. luminesce in the energy range of $1.6-2.5 \mathrm{eV}$ whereas, the PL in PS in this range increased with etching time. This PL was fitted to a Gaussian line shape along with an exponential background. The PL peak position, full width at half maximum and area are tabulated in table 1. This $\mathrm{PL}$ is assigned to $\mathrm{S}$ band (Cullis et al 1997) and its peak position blue-shifts from $2 \cdot 1-2 \cdot 3 \mathrm{eV}$ as etching time increases from $30-90 \mathrm{~min}$. The PL peak broadens a little as the etching time increases. The intensity increases by a factor of about 55 on increasing the etching time from 30 to $90 \mathrm{~min}$, which results in the decrease of diameter from $2 \cdot 9-2 \cdot 3 \mathrm{~nm}$. In $\S 3 \cdot 3$, it was suggested that the disordered Si region around the pores in the 30 min etched PS would have dissolved on etching further to $90 \mathrm{~min}$. The disordered $\mathrm{Si}$ region around the pores in $30 \mathrm{~min}$ PS might have had a large number of non-radiative centres resulting in weak PL. On dissolving the disordered Si region in

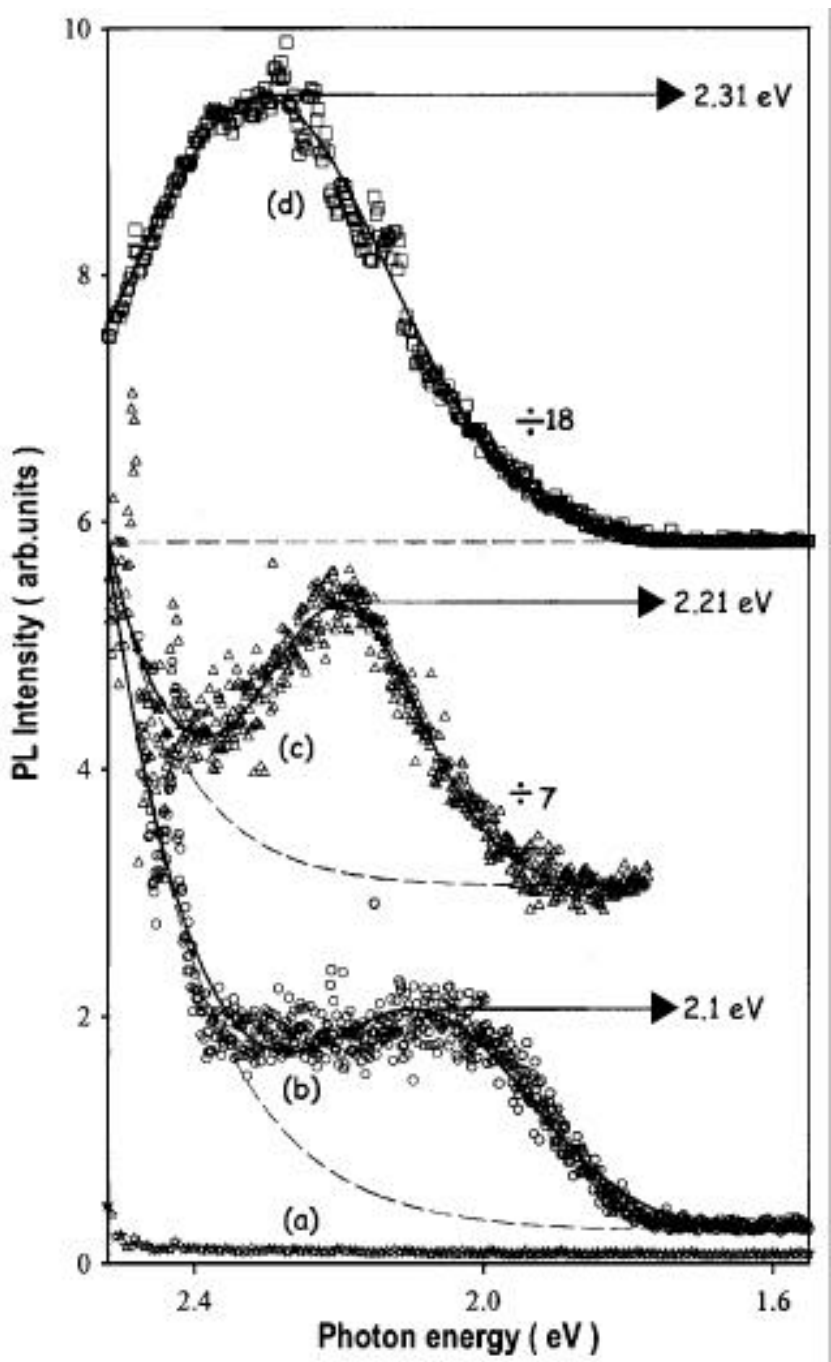

Figure 4. Photoluminescence spectra of (a) $c$-Si, (b) 30, (c) 60 and (d) 90 min etched PS samples. The solid lines are the fit to a Gaussian lineshape and an exponential background shown as broken line. The curves are shifted along the intensity axis for clarity. 
90 min PS, the PL increased by a factor of 55 . There is another reason for the phenomenal increase in PL intensity by a factor of 55 on increasing the etching time from 30-90 min. As the Si nanocrystallite becomes thinner and unsupportive with disordered $\mathrm{Si}$ or $a-\mathrm{Si}$, the strain in nanocrystallite could anneal the defects in the crystallite. Some point defects and dislocations are known centres of non-radiative recombination. Annealing them would increase the PL intensity.

The observed S-band PL emission in PS samples is due to the established fact of quantum confinement of electrons in the nanosized particles of $c$-Si present in PS (Bisi et al 2000). The blue shift in the PL peak position implies a reduction in the diameter of the Si nanocrystallite size with increase of etching time. In addition, it also gives strong evidence for the band gap widening of the PS samples.

Figure 5 shows the experimental and theoretical optical absorption and photoluminescence peak positions in PS as a function of the Si nanocrystallite diameter using electron confinement model (Lannoo et al 1997). The continuous curve is the size dependence of the theoretical optical absorption peak position in PS nanocrystallites. The broken curve is that of the photoluminescence peak position in PS after the excitonic correction to the absorption curve. The open and filled symbols are the experimental absorption and emission position, respectively. It is noted that the experimental optical absorption peak

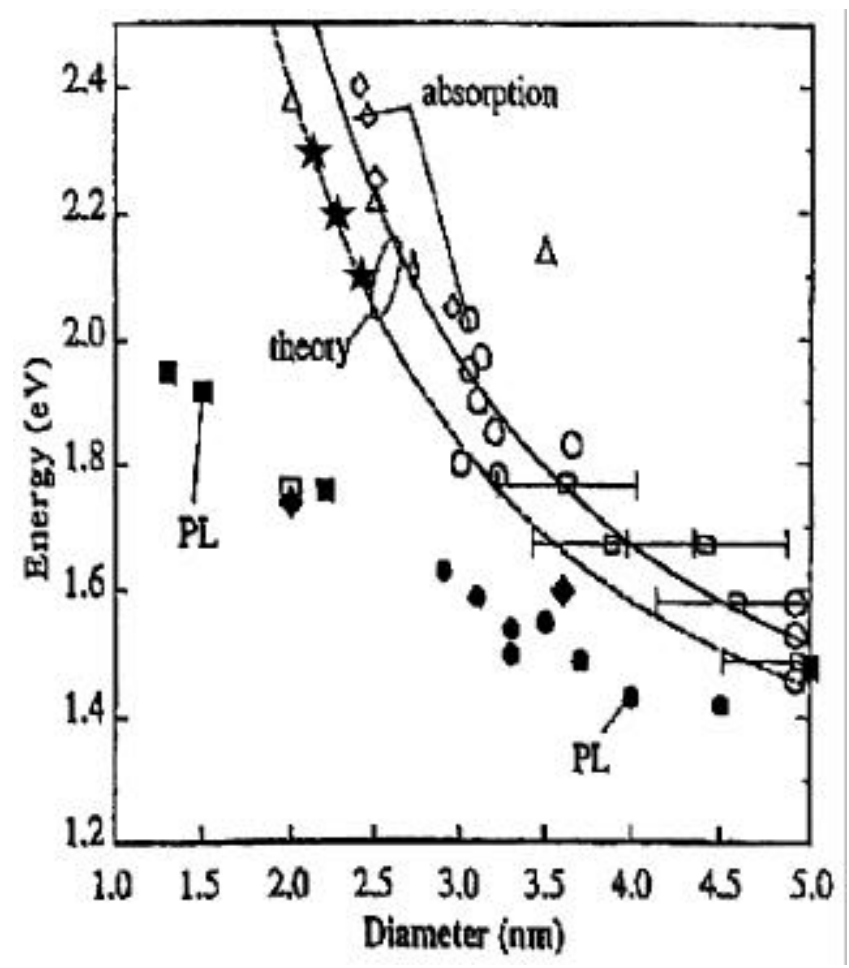

Figure 5. The band gap vs Si nanocrystallite diameter plot. Theoretical curves for optical absorption (solid line) and photoluminescence (broken line) are shown. The present data $(\star)$ and other data are shown for comparison. positions match well with the theory while the experimental emission peak positions lie well below the calculated values. Siloxene derivatives and $\mathrm{SiH}_{x}$ kind of chemical species would give luminescence in the visible range (Bisi et al 2000). This will affect PL from Si nanocrystallites. Since our PS samples do not have such chemical species, PL intensity is dominantly from the $\mathrm{Si}$ nanocrystallites. Filled star symbols on the theoretical PL curve of figure 5 are the present experimental values of the PL peak positions for 30, 60 and $90 \mathrm{~min}$ PS, respectively. The shape of the $\mathrm{Si}$ nanocrystallite is not spherical, as shown in figure 1 . The electron confinement model (Lannoo et al 1997) and the phonon confinement model (Xu et al 1999) used here are not sensitive enough to the deviations from a spherical shape. The effective average diameter of the spherical $\mathrm{Si}$ nanocrystallites has been estimated in $\$ 3.2$. The same is estimated using the PL also. The size of the $\mathrm{Si}$ nanocrystallites as determined from our experimental PL peak position using the broken curve of figure 5 based on the electron confinement model is $2 \cdot 4,2 \cdot 3$ and $2 \cdot 14 \mathrm{~nm}$ for 30,60 and $90 \mathrm{~min}$ etched PS, respectively (Lannoo et al 1997). They are given in table 1 . The corresponding Si crystallites size as determined from Raman spectra based on phonon confinement is $2.9,2.6$ and $2.3 \mathrm{~nm}$. The size estimated from the electron confinement is lower than that from phonon confinement. However, the magnitude of the size and the trend of size reduction on increasing etching time are the same. It is known that PL peak position blueshifts for the same Si nanocrystallite when its temperature is increased (Cullis et al 1997). We have measured the temperature of the PS surfaces during PL measurement with laser excitation as $50^{\circ} \mathrm{C}$ for $c-\mathrm{Si}, 500^{\circ} \mathrm{C}$ for $30 \mathrm{~min} \mathrm{PS}, 700^{\circ} \mathrm{C}$ for $60 \mathrm{~min}$ PS and $1000^{\circ} \mathrm{C}$ for $90 \mathrm{~min}$ PS by recording the stokes and antistokes Raman spectra of LO phonon and equating the area ratio to the Boltzman factor (Prabakaran 2002). The temperature correction to PL peak position would result in increased Si nanocrystallite size that matches well with the Raman estimate.

\section{Conclusions}

The red shift in the LO phonon Raman spectra and the blue shift in photoluminescence spectra reveal the quantum confinement effect in the Si nanocrystallites of the PS sample. As the etching time increases, these shifts increase due to decrease in the silicon nanocrystallite size. This is indicated by the decrease in the estimated diameter of spherical nanocrystallites from $2.9-2.3 \mathrm{~nm}$ using the Raman spectra. Presence of these Si nanocrystallites has been confirmed from the HRTEM measurement. Similar sizes for the PS samples have been obtained from PL also. The photoluminescence showed an increase of PL intensity by a factor of 55 in 90 min PS as compared to that in $30 \mathrm{~min}$ PS, for a nominal change of Si nanocrystallite size $(2.3 \mathrm{~nm}$ for $90 \mathrm{~min}$ PS and $2.9 \mathrm{~nm}$ 
for $30 \mathrm{~min}$ PS). It is suggested that it might be due to the dissolution of the disordered Si region present in $30 \mathrm{~min}$ PS for 90 min etching. This is supported by $2 \mathrm{TO}$ mode intensity.

\section{References}

Berbezier I and Halimaoui A 1993 J. Appl. Phys. 745421

Bisi O, Stefano Ossicini and Pavesi L 2000 Surf. Sci. Rep. 381

Campbell I H and Fauchet P M 1986 Solid State Commun. 58 739

Canham L T 1990 Appl. Phys. Lett. 571046

Canham L T 1992 Phys. World 541

Cullis A G and Canham L T 1991 Nature 353335

Cullis A G, Canham L T and Calcott P D J 1997 Appl. Phys. Rev. 82909

Edelberg Erik, Bergh Sam, Naone Ryan, Hall Michael and Aydil Eray S 1997 J. Appl. Phys. 812410

Gregora I, Champagnon B and Halimaoui A 1994 J. Appl. Phys. 753034

Lannoo M, Allan G and Delerue C 1997 Structural and optical properties of porous silicon nanostructures (eds) G Amato, $\mathrm{C}$ Delerue and $\mathrm{H} \mathrm{J}$ von Berdeleben (Amsterdam: Gordon and Breach) p. 187

Lee H J et al 1995 Appl. Phys. Lett. 62855

Prabakaran R, Raghavan G, Tripura Sundari S, Kesavamoorthy R and Xavier F P 2002 Physica E15 243

Prabakaran R, Kesavamoorthy R, Amirthapandian S and Xavier F P 2003 Physica B337 36

Prabakaran R, Kesavamoorthy R, Amirthapandian S and Ramanand A 2004 Mater. Letts 583745

Prabakaran R, Raghavan G, Tripura Sundari S and Kesavamoorthy R 2005 Solid State Commun. 133801

Richter H, Wang Z P and Ley L 1981 Solid State Commun. 39 625

Sood A K, Jayaram K, Victor D and Muthu S 1992 J. Appl. Phys. 724963

Sui Zhifeng, Leong Patrick P and Herman Irving P 1992 Appl. Phys. Lett. 602086

Tubino R, Piseri L and Zerbi G 1972 J. Chem. Phys. 561022

Xu Dongsheng, Guo Guolin, Gui Linlin, Tang Yougi, Zhang B R and Qin G G 1999 J. Appl. Phys. 862066

Yoffe A D 1993 Adv. Phys. 42173 